\title{
GRUPO DE ACOLHIDA EM SAÚDE MENTAL: A PSICOLOGIA NAATENÇÃO BÁSICA
}

\author{
RECEPTION GROUP IN MENTAL \\ HEALTH: PSHYCOLOGY IN \\ PRIMARY HEALTH CARE
}

Luiza Maria de Oliveira Braga Silveira*

Luciana Suárez Grzybowski**

Roberta da Silva Gomes ${ }^{* * *}$

Gabriela Brito Pires ${ }^{* * * *}$

Thamy de Oliveira Azambuja*****

Fernanda Anderle

\section{RESUMO}

O presente artigo vem compartilhar a experiência de um grupo de acolhida em saúde mental na atenção básica realizada a partir do projeto de extensão "Grupos de acolhida na Saúde Mental como dispositivo de atenção à saúde mental e de integração do Serviço-Escola do Curso de Psicologia da UFCSPA à rede de atenção básica", da Universidade Federal de Ciências da Saúde, de Porto Alegre, RS. Constitui-se como um grupo aberto, existente desde 2012, com o objetivo de dar suporte psicológico aos pacientes atendidos em Unidades Básicas de Saúde que fazem parte da região docente-assistencial dessa universidade. A efetividade deste grupo foi constatada através da melhora clínica e interpessoal dos seus participantes. Concluiu-se que, além de promover a integração ensino-serviço, os acolhimentos em saúde mental na atenção básica são necessários, considerando-se de extrema importância a realização de grupos de acolhida em saúde mental em outras Unidades de Saúde da região.

Palavras-chave: acolhida; saúde mental; atenção básica; grupos.

\footnotetext{
* Professora da Universidade Federal de Ciências da Saúde de Porto Alegre (UFCSPA), RS - Brasil. E-mail: luizasilveira@hotmail.com

** Professora da Universidade Federal de Ciências da Saúde de Porto Alegre (UFCSPA), RS - Brasil. E-mail: luciana.sgrzybowski@ gmail.com

*** Psicóloga. E-mail: robertadasg@gmail.com

**** Psicóloga. E-mail: gabrielabppsi@gmail.com

***** Aluna de graduação da Universidade Federal de Ciências da Saúde de Porto Alegre (UFCSPA), RS - Brasil. E-mail: thamy.az@ gmail.com
}

****** Aluna de graduação da Universidade Federal de Ciências da Saúde de Porto Alegre (UFCSPA), RS - Brasil. E-mail: anderle. fernanda@yahoo.com.br 


\section{ABSTRACT}

This article aims atsharing the experience of a primary mental health care reception group, which is an extension practice from the project "Reception groups in Mental Health as a device of mental health attention and integration of teaching service of UFCSPA Psychology Course with the primary health care network" from the Federal University of Health Sciences of Porto Alegre (UFCSPA). It is an open group, which has been in existence since 2012. Its objective is to provide psychological support to patients treated in Primary Health Services that are part of the teaching-service area of UFCSPA. The effectiveness of this group was observed trough clinical and interpersonal improvement of its participants. The conclusion points to the fact that in addition to promoting teaching service integration, mental health welcoming is needed in primary health attention, considering the extreme importance of receptions groups in mental health in other health services of the region.

Keywords: welcoming; mental health; primary health care; groups.

\section{Introduçáo}

O Sistema Único de Saúde (SUS) foi criado em 1990, através da Lei no 8.080, que versa sobre as condiçóes para a promoção, proteção e recuperação da saúde em nosso país (BRASIL, 1990). A Constituição de 1988, no artigo 198, determina a saúde como direito universal, e o Estado tem como dever proporcionar o seu acesso ao cidadão (BRASIL, 1988). Os princípios que regem o SUS são: universalidade, equidade, participação da comunidade, resolutividade e gratuidade dos serviços a toda a população (BRASIL, 1988). Para sua organização, também seguemas seguintes diretrizes: descentralização, atendimento integral e participação popular (FONTOURA;MAYER, 2006)

A atençáo básica $(\mathrm{AB})$ é o nível de complexidade em saúde na qual se encontra a principal porta de entrada para o SUS, por meio da Unidade Básica de Saúde (UBS) e da Estratégia de Saúde da Família (ESF). Com o objetivo de colocar em prática os princípios e as diretrizes propostos pelo SUS, a $\mathrm{AB}$ caracteriza-se por ser um conjunto de açóes, no âmbito individual e coletivo, que abrange a promoçáo e a proteção da saúde, a prevenção de agravos, o diagnóstico, o tratamento, a reabilitação, a redução de danos, além de sua manutenção. A AB busca o desenvolvimento de uma atenção integral que contribua para melhora na situação de saúde e autonomia das pessoas, em umnível de atenção primário à saúde (BRASIL, 2012).

Apesar desta proposição, o SUS ainda tem desafios a enfrentar para consolidar seus princípios e avançar como política pública acessível e resolutiva. Entre eles, são destaques:o financiamento insuficiente do setor, a precarização do trabalho, os modos de se produzir a atenção aos usuários e a gestão da saúde pública. Para além dessas questóes, a formação dos trabalhadores que atuam na $\mathrm{AB}$ ainda aponta carências quanto às habilidades necessárias para a realização de um atendimento mais humanizado ao usuário, especialmente na área de saúde mental. Isto tende a interferir na solução das demandas (NEVES; LUCCHESE; MUNARI, 2010; SOARES, 2008; CARNEIRO et al., 2009), pois estas dificuldades incidem sobre os profissionais náo-familiarizados com a área de saúde mental, não se sentindo capacitados para atender ou sequer acolher os usuários com este tipo de demanda (DIMENSTEIN et al., 2009). Por isso, reforça-se a ideia de que o único atendimento possível é um atendimento especializado.

Assim, a Política Nacional de Humanização (PNH) aponta que alguns modos de organização dos processos de trabalho em saúde facilitam o enfrentamento dos problemas e potencializam a existência de saídas criativas e singulares para cada contexto. Umas das diretrizes propostas pela $\mathrm{PNH}$, no que tange a melhora da atençáo e assistência em saúde, relaciona-se com o acolhimento (BRASIL, 2010). 
O acolhimento é uma diretriz que visa possibilitar o atendimento integral à pessoa que busca o serviço de saúde (BRASIL, 2010). Pode ser entendido como conteúdo de toda atividade assistencial, abrangendo a busca de um reconhecimento cada vez maior das necessidades de saúde dos usuários e das formas de satisfazê-las. Tal prática tem se mostrado eficiente para a realização de atendimentos e encaminhamentospela rede assistencial, levando-se em consideração as necessidades e as possibilidades de cada usuário (TEIXEIRA, 2003).

O Ministério da Saúde entende o acolhimento como uma tecnologia de relação presente nos encontros, em que há o reconhecimento e a aproximação com o outro (BRASIL, 2004). Outros autores (AZAMBUJA et al., 2007; FRANCO; BUENO; MERHY, 1999; CARDOSO; PEREIRA, 2010) são unânimes em afirmar que o acolhimento difere da triagem, a qual consiste em encaminhar o usuário de acordo com a sua demanda, ao passo que o acolhimento consisteem um instrumento de escuta e responsabilização do profissional para com o usuário, de forma a garantir acesso universal, humanizado e integral (FRANCO et al., 1999). Possibilita, ainda, a adequação técnica e assistencial dos profissionais e dos serviços de saúde à clientela (AZAMBUJA et al., 2007; FRANCO et al., 1999), estabelecendo reflexão e mudanças nas relaçóes entre a equipe e sua lógica de trabalho (FRANCO et al., 1999).

Considerando a saúde mental uma das atribuiçốes da atenção básica, Jorge et al. (2011) falam a respeito da integralidade e da humanizaçáo do cuidado, aprofundando as relaçóes subjetivas entre trabalhador/usuário/serviço de saúde através de práticas fundamentadas no acolhimento, no diálogo, no vínculo, na corresponsabilidade e na escuta ativa. Dessa forma, o acolhimento articulado ao estabelecimento de vínculo entre usuário e trabalhadores da saúde contribui com cuidado integral em saúde mental, transpondo a patologia e o diagnóstico e ressaltando a subjetividade e singularidade de cada um, facilitando, dessa maneira, a construção da autonomia mediante responsabilização compartilhada e pactuada entre os sujeitos envolvidos (BRASIL, 2010; COSTA, 2004;JORGE et al., 2011).

Uma das práticas fundamentais em saúde preconizada nas ações da atenção básica é a realização de grupos. Esse tipo de intervenção permite organizar os processos de trabalho e ampliar a capacidade assistenciale, por vezes, a qualidade do atendimento ao usuário (CHIAVERINI, 2001). Alguns dos benefícios do atendimento em grupo são o estabelecimento de identificações, a reprodução de conflitos, o desenvolvimento de novas formas de relacionamento, espaços importantes de apoio social e aprendizagem interpessoal (ZIMERMAN; OSÓRIO,1997).

O grupo, como um sistema complexo, constitui um método efetivo para acolher, refletir e desenvolver uma aprendizagem para responder à complexidade das relaçóes humanas contemporâneas (SEMINOTTI, 2016a).O grupo de acolhida em saúde mental explora o potencial terapêutico dos seus participantes, e a força para a mudança acaba por decorrer da interação entre os seus membros (GUANAES;JAPUR, 2001; GUIMARÁES, 2001; BECHELLI;SANTOS, 2001, 2002). Sendo assim, o fator terapêutico é gerado pelo grupo e contribui para a melhoria da condiçáo de cada um e do grupo como unidade.

Segundo o Guia de Matriciamento (CHIAVERINI, 2001), os grupos da atençáo básica devem ter cunho educativo e também proporcionar reflexão e suporte aos participantes. Um grupo de acolhida com foco na saúde mental dá conta de vários princípios, diretrizes e recomendaçóes propostas pela Política Nacional de Atenção 
Básica (PNAB). Essa intervenção auxilia na minimização de dificuldades psicossociais que impedem o desenvolvimento integral das pessoas da comunidade, mostrando-se como um espaço para escuta e troca de vivências entre os participantes. A prática do grupo de acolhida tem como foco uma concepçáo ampliada de saúde, indo ao encontro da integralidade e da responsabilidade compartilhada no cuidado. Objetiva a construçáo de conhecimentos em coletividade, buscando fortalecer o usuário como protagonista das suas ações no serviço de saúde, fundamentando-se nos pressupostos da educação popular, processos grupais e análise institucional (AZAMBUJA et al., 2007).

Destaca-se, assim, a importância de tais grupos reconhecerem os saberes e poderes dos indivíduos que trazem a queixa e a problemática da saúde mental em seu contexto, em suas vidas. Uma vez que os grupos reproduzem a hierarquia social e são capturados por ela, podem tender a pouco valorizar o saber e o protagonismo popular, como as demais práticas de saúde, conduzindo a uma passividade e constante subtração de recursos e valores em saúde e educação. Já ao inserir distintos saberes e realidades e discuti-los coletivamente, pode proporcionar uma abundância de recursos. Isso porque multiplicamse náo apenas os conhecimentos, mas os atores e os equipamentos sociais, a saber todos os profissionais e usuários da saúde (SEMINOTTI, 2016a).

Desde a legislaçáo do SUS, preconiza-se a realizaçáo de atividades coletivas como forma de atenção aos usuários e de fortalecimento dos vínculos entre os usuários, os trabalhadores e os usuários e, também, dos usuários com a promoçáo da saúde (SEMINOTTI, 2016b). Assim, os grupos de acolhida também se mostram como uma forma de fortalecer capacidades individuais e coletivas. Objetiva-se a potencializaçáo de recursos pessoais e grupais para o enfrentamento dos determinantes de saúde e de doença, atingindo mais do que apenas o conhecimento sobre fatores de risco e proteçáo de determinados quadros de doenças (CZERESNIA, 2003).

A partir destas questóes, o presente artigo vem compartilhar a experiência de realização de um grupo de acolhida em saúde mental no contexto da atenção básica, que faz parte do projeto de extensão "Grupos de acolhida na Saúde Mental como dispositivo de atenção à saúde mental e de integração do Serviço-escola do Curso de Psicologia da UFCSPA à rede de atençáo básica”, da Universidade Federal de Ciências da Saúde de Porto Alegre (UFCSPA).

Este projeto tem como um de seus objetivos dar suporte psicológico aos pacientes atendidos em Unidades Básicas de Saúde(UBS)que fazem parte da região docenteassistencial na qual se insere o Serviço-Escola do Curso de Psicologia da UFCSPA. Portanto, o grupo de acolhida em saúde mental está inserido no contexto da Atençáa Básica, em uma Unidade Básica de Saúde de Porto Alegre/RS. Essa ação, vinculada ao Serviço-Escola de Psicologia da UFCSPA, integra as ênfases propostas no curso de Psicologia dessa universidade (Processos de Promoção e Prevenção em Saúde e Processos de Gestão em Saúde Mental Coletiva), demonstrando a indissociabilidade ensinopesquisa-extensão.

A integração ensino-serviço é citada dentre as inovaçōes pedagógicas no processo ensino-aprendizagem dos profissionais em saúde e evidencia a integração educaçáo e saúde, nas políticas de formação e atuação. Tal integração objetiva garantir ações e serviços de qualidade à população, através de cenários e práticas diversos que proporcionam aprendizagem, reflexão, criação, proposição e construção de um novo modelo de cuidado 
em saúde. Assim, retroalimentam-se: o ensino (e a formação profissional) e prática em serviço (KUBARA et al., 2014; VIEIRAet al., 2016).

Sabe-se que para efetivar os propósitos relativos à formação acadêmica em saúde e à integraçáo da Universidade com a comunidade externa, é preciso que as faculdades tenham campos de ação que possibilitem a seus discentes entrar em contato com a realidade social e colocar em prática seus aprendizados, aprendendo e inovando os já consolidados em ambos campos (BALDOINO, VERAS, 2016). Assim, a realização do grupo de acolhida em saúde mental vem atendendo uma demanda importante da regiáo docente-assistencial na qual se insere,bem como de aprendizado dos alunos do Curso de Psicologia, através do seu Serviço - escola.

Dessa forma, também se desenvolve o conhecimento, o alcance e o aprimoramento das práticas psicológicas no campo da atenção básica em saúde, ampliando a inserção e as açóes do Serviço em questão.

O grupo tem ocorrido desde 2012 e vem consolidando seu espaço como dispositivo da rede de atenção em saúde mental da Gerência Distrital Norte-Eixo Baltazar (GDNEB), que compóe a regiáo norte da cidade, parte do Distrito Docente Assistencial. A vivência dos bolsistas do projeto tem se mostrado como diferencial em suas práticas de estágio curriculares e eventos acadêmicos, revelando também sua vinculação ao ensino e à pesquisa, e à integração ensino-serviço.

\section{Metodologia}

Este artigo tem o objetivo de descrever um relato de experiência acerca de um projeto de extensão, cujo objetivo é aprimorar, planejar, implementar, qualificar e avaliar a realização de um grupo de acolhida em saúde mental em uma UBS inserida no Distrito Docente-Assistencial da UFCSPA. A atuação se dá junto ao Serviço-Escola do Curso de Psicologia da UFCSPA e à gerência distrital de saúde de sua região de abrangência.

Através de editais internos de extensão, o projeto tem sido submetido pelas coordenadoras com a anuência do Núcleo de Integração Ensino-Serviço (NIES), que é uma parceria da Universidade com a Secretaria Municipal de Saúde, em seu Distrito Docente Assistencial. Assim, anualmente, é avaliado, aprovado e formalizado pela comissão de extensão da Universidade, contemplando-o com um bolsista institucional, além de alunos voluntários. Os alunos que participam do projeto de extensão são acadêmicos do curso de Psicologia, cursando entre o $5^{\circ}$ e o $10^{\circ}$ semestre. $\mathrm{O}$ projeto possui aprovação e financiamento da Pró-Reitoria de Extensão, através de editais anuais.

A metodologia de encaminhamento de usuários ao grupo ocorre através de discussão dos casos que foram previamente trazidos no matriciamento em saúde mental. Semanalmente, ocorrem as reunióes do Serviço-Escola, nas quais os estagiários do curso de Psicologia, que integram os encontros de matriciamento realizados pelo NASF da região, relatam as demandas discutidas, e, conjuntamente, a equipe reflete sobre o melhor encaminhamento para cada caso. Se é acordado o benefício de participação no grupo, os alunos entram em contato com o usuário para realizar o convite. Outras formas de ingresso no grupo se dáo através de encaminhamentos diretos tanto por parte da equipe de matriciamento em saúde mental, quanto por trabalhadores das unidades de saúde da região. 
Quando há o ingresso de um novo participante, os alunos que coordenam o grupo realizam uma acolhida inicial individual, a fim de conhecer a real demanda do indivíduo, bem como explicar o funcionamento do espaço, proporcionando que o sujeito se sinta à vontade e pertencente ao grupo. Além disso, existe também a possibilidade de ser realizada uma acolhida final individual com alguns participantes. Isto ocorre naqueles casos em que o sujeito está muito mobilizado com alguma situação abordada no dia, ou alguma outra demanda específica. A acolhida final tem por objetivo tranquilizar o participante, demonstrando que o grupo é capaz de acolher as suas angústias e demandas, das formas mais variadas possíveis.

O grupo tem frequência semanal e os encontros têm duração média de uma hora. Atualmente, um aluno bolsista de extensáo e um aluno voluntário coordenam o grupo. Sua prática está fundamentada nos grupos operativos de Pichon-Rivière (2005). O trabalho com grupos operativos propóe em sua técnica um processo de aprendizagem para os sujeitos envolvidos. Os grupos operativos sempre visam "operar" em uma determinada tarefa - a aprendizagem. Tendo um método não-diretivo, a técnica do Grupo Operativo transforma uma situação de grupo em um espaço de investigação-ativa (ZIMERMAN; OSÓRIO, 1997; PICHON-RIVIÈRE, 2005). Para isso, o coordenador tem a função de facilitar a comunicaçáo entre os integrantes, a fim de que o grupo seja operativo; isto é, que ultrapasse os obstáculos na resolução da tarefa.

Ao final de cada encontro, os alunos relatam o grupo em ata formal, que serve de material de análise (tanto teórica, como prática) da equipe. Também são realizadas semanalmente supervisóes com a professora orientadora do projeto, espaço que tem por objetivo analisar a atuação das alunas enquanto terapeutas, pensando coletivamente nas intervençôes técnicas utilizadas, e buscando aprimorá-las a cada nova experiência. Além disso, busca-se refletir acerca dos fenômenos e processos grupais neste contexto específico da acolhida e das práticas em saúde pública, particularmente na atenção básica.

\section{Resultados e Discussáo}

O grupo de acolhida em saúde mental desenvolvido constitui-se como um grupo aberto, com distintos participantes a cada encontro e ocorre desde setembro de 2012. Os grupos são caracterizados pela definição de objetivos realistas e específicos, por uma relativa homogeneidade entre os participantes e por posturas mais ativas por parte do coordenador, através do oferecimento de conselhos, sugestóes e apoio (VINOGRADOV;YALOM, 1992; SALVENDY, 1996).

Ao longo de quatro anos de existência, o grupo manteve-se ininterrupto, oscilando em relaçáo ao número de participantes, porém nunca esteve sem um usuário. Houve, ao longo destes anos, um predomínio de mulheres. Desde o começo do grupo, em 2012, participaram do espaço 96 usuários, sendo um total de 81 mulheres $(84,37 \%)$ e 15 homens (15,65\%), em sua maioria com idades acima de 40 anos. Os participantes revelam compreender o grupo como um local no qual sentem-se seguros, compreendidos e isentos de julgamentos, tendo, dessa forma, as suas demandas acolhidas.

Neste sentido, observou-se que as principais demandas entre os usuários forama existência de quadros depressivos $(56,25 \%)$ e/ou de ansiedade (10,41\%), e também foi possível observar que a maioria $(51,04 \%)$ dos usuários fez/faz uso de psicofármacos. Os 
transtornos depressivos, em estudos de base populacional, atingem prevalência de $10 \%$ e incidência de $2 \%$ na população. Apesar dos índices não serem comparáveis ao da população geral (pois são usuários encaminhados ao serviço de saúde mental), a prevalência pode ser considerada alta, independentemente do local do estudo, do instrumento utilizado e dos períodos de tempo para os quais a prevalência se aplica (ROMBALDI et al, 2010).

Além disso, achados na literatura mostram a dificuldade de comprometimento para autocuidado e manutenção do tratamento medicamentoso, cuja interrupção ou utilizaçáo de forma inadequada pode ter os sintomas intensificados, iniciando um possível ciclo vicioso (IBANEZ et al., 2014). Neste cenário, o grupo surge como uma alternativa para auxiliar e compreender o uso de taismedicamentos, o que nem sempre é bem esclarecido pela equipe de saúde.

Quanto à frequência de participação no grupo, a média de comparecimento dos usuários é de 7 encontros. Dos 15 homens, apenas 1 participou de 10 encontros, enquanto os outros tiveram menos de 5 participações. Já em relação às mulheres, 1 está participando do grupo desde o seu início, 2 participam há 3 anos e 1 participa há 2 anos. A adesão aesta atividade reflete a sua importância como profilaxia em saúde mental. No ano de 2016, nenhum homem participou do grupo até o mês de junho, corroborando com a literatura, que indica a dificuldade do acesso dos homens ao serviço de atenção primária à saúde (FIGUEIREDO, 2005), ao passo que as mulheres buscam com mais frequência os serviços de saúde.

Destaca-se que suas demandas também se relacionam ao auxílio em questóes decorrentes de contexto social (COELHO et al., 2009), as quais parecem influenciar também a saúde mental das mulheres, como é visto com frequência no Grupo de Acolhida. Além disso, ao longo destes 4 anos de realização do grupo, esse passou a configurar-se como um grupo de mulheres, o que dificulta os encaminhamentos de homens através dos matriciamentos, assim como a permanência dos mesmos quando chegam ao grupo, possivelmente pela falta de identificação nas demandas e nas questóes de gênero.

Os participantes fazem questionamentos sobre as alternativas de apoio e suporte emocional, mostrando-se protagonistas de sua melhora. A consolidação da identidade de grupo mostra-se importante fator para o desenvolvimento dos encontros e adesáo dos participantes. É possível verificar o crescimento da empatia para acolher as demandas do outro, tornando o processo terapêutico cada vez mais eficaz para a totalidade grupal (PINHEIRO et al., 2002; COELHO et al., 2009). Dessa forma, explicita-se que os principais objetivos do grupo estão sendo atingidos, reassegurando sua função junto aos usuários e suas demandas individuais e coletivas.

Constatou-se uma melhora clínica e interpessoal dos participantes do grupo, com consequente empoderamento individual, familiar, social e comunitário. Seus objetivos parecem estar sendo alcançados, uma vez que visa construir a capacidade dos indivíduos em tomar suas próprias decisões, para que possam buscar por melhores condições de vida, bem como possuir um controle maior sobre a situação de exclusão social. $\mathrm{O}$ empoderamento individual e coletivo e a participação comunitária são elementos fundamentais para a promoção de saúde (VIEIRA etal., 2016). A reduçáo ou a própria adesão ao uso de medicação, a ampliação da vida social e comunitária, a melhoria das relaçóes familiares e o aumento da autonomia são exemplos dessas mudanças observadas.

O empoderamentopode ser observado através dos questionamentos sobre as alternativas de apoio e suporte emocional feitos durante os grupos, mostrando os 
participantes como protagonistas de sua melhora. Observa-se também esse fenômeno em nível grupal, evidenciado através do respeito entre os membros, do apoio mútuo e da busca por objetivos idealizados e comuns entre estes. Essas transformaçóes parecem possibilitar o sentimento de pertencimento, de práticas solidárias e de reciprocidade, indicado através da consolidação da identidade de grupo, cujo papel é visto como importante fator para o desenvolvimento dos encontros e adesão dos participantes (KLEBA; WENDHAUSEN,2009).

A existência, há 4 anos, deste grupo de acolhida representa uma grande conquista para a rede de saúde mental do território em que se insere. De acordo com as análises e avaliaçóes realizadas pelos usuários, fica evidente a sua importância na promoção e prevençáo à saúde, principalmente por se tratar de um importante dispositivo de atençáo à saúde de modo geral. Da mesma forma, a permanência do grupo cumpre com um papel relevante na formaçáo dos alunos no que diz respeito ao contexto da saúdemental e pública, bem como na atenção básica.

\section{Conclusóes}

Observa-se que o entrelaçamento teoria e prática, através das práticas em extensão vinculadas ao Serviço-Escola de Psicologia, além de contribuir para a formação dos alunos em formação, faz com que a instituição cumpra com o seu papel social (SALINAS;SANTOS, 2002). Neste contexto, a utilizaçáo de grupos operativos surge como um dispositivo capaz de consolidar uma concepçáo do homem em sua integralidade, para além do foco de compreensão saúde-doença, visando uma formação em saúde mais reflexiva, integrada e humanizada (SILVEIRA;RIBEIRO, 2005).

$\mathrm{O}$ aprendizado e as mudanças se mostram presentes nos participantes, com o aumento de sua autonomia e com a compreensão da vivência no grupo como um fator de fortalecimento para lidarem com dificuldades que enfrentam em sua rotina. A participação e a vinculação nos grupos também contribuempara o aumento da reflexáo e da empatia dos participantes. Desta forma, o grupo auxilia no processo de oparticipante tornar-se consciente de si, de seu contexto e de suas escolhas.

O território em que a ação é realizada é caracterizado por ser uma regiáo vulnerável da cidade de Porto Alegre, na qual não há psicólogos na $\mathrm{AB}$ para o atendimento à populaçáo adulta. Assim sendo, considerando o difícil acesso a espaços que promovam a saúde mental na $A B$ no contexto descrito, o grupo se constituiu como um importante dispositivo de saúde mental na $\mathrm{AB}$, possibilitando o fortalecimento do vínculo dos usuários com o serviço, realizando uma escuta qualificada e promovendo o cuidado integral à saúde. Através do acolhimento, diferentes processos podem ser operados, e o grupo de acolhida em saúde mental busca minimizar problemas psicossociais que impedem o desenvolvimento humano integral da comunidade referenciada, permitindo um espaço para escuta e troca de vivências (SILVEIRA et al.,2014).

Compreende-se que este projeto possui limitaçôes no que diz respeito à disparidade na adesão dos participantes, havendo um número superior de participantes mulheres em relação aos homens, ao longo dos anos de sua realização. Dessa forma, tem sido cuidado para que não seja caracterizado como um grupo de acolhida para mulheres. Tal fato pode revelar-se como um indicativo às dificuldades do acesso dos homens aos serviços de saúde. 
Desta forma, acredita-se na importância de buscar por dispositivos para a desconstrução dessa caracterização grupal e que possibilitem uma maior participação e consequente adesão de homens ao grupo de acolhida.

Tendo em vista a efetividade deste grupo de acolhida, a carência de psicólogos na região na qual está inserido o Serviço-Escola, bem como a necessidade de acolhimentos em saúde mental na atenção básica, de acordo com as preconizaçóes da PNAB (2010), considera-se de extrema relevância a realização de grupos de acolhida em saúde mental em outras Unidades de Saúde da regiáo, proporcionando o aumento dos atendimentos em saúde mental prestados à população adulta deste território, além de uma maior possibilidade de atuação e qualificação dos estudantes em formação.

\section{Referências}

AZAMBUJA, M. P. R.; DEBASTIANI, C.; DUARTE, C. C.; MINOZZO, F.; SOUZA, A. C. Relato de experiência: $O$ acolhimento em grupo como uma estratégia para a integralidade. PsicoUSF, v.12, n.1, p. 121-124, 2007.

BALDOINO, A. S.; VERAS, R. M. Análise das atividades de integração ensino-serviço desenvolvidas nos cursos de saúde da Universidade Federal da Bahia. Revista da Escola de Enfermagem,v.50, n.1, p. 17-24,2016.

BECHELLI, L. P. C.; SANTOS, M. A. Psicoterapia de Grupo: noções básicas. Ribeirão Preto, SP: Legis Summa, 2001.

BECHELLI, L. P. C.; SANTOS, M. A. Psicoterapia de grupo e considerações sobre o paciente como agente da própria mudança. Revista Latino-Americana de Enfermagem, Ribeirão Preto, v.10, p. 383-391, 2002.

BRASIL. Lei n 8.080, de 19 de setembro de 1990: Dispõe sobre as condições para a promoção, proteção e recuperação da saúde, a organização e o funcionamento dos serviços correspondentes e dá outras providências. Diário Oficial da união, Brasília, DF, 20 set.1990. Disponível em: <http://www.planalto.gov.br/ccivil 03/Leis/L8080.htm>. Acesso em: 16 jul. 2016.

BRASIL. Constituição de 1988. Constituição da República Federativa do Brasil. 18.ed. Brasília, DF: Senado Federal, 1988.

BRASIL. Ministério da Saúde. Política Nacional de Atenção Básica. Brasília: Ministério da Saúde, 2012. (Série E. Legislação em Saúde). Disponível em: <http://189.28.128.100/dab/ docs/publicacoes/geral/pnab.pdf>. Acesso em: 16 jul. 2016.

BRASIL. Ministério da Saúde. Secretaria de Atenção à Saúde. HumanizaSUS:Documento Base para Gestores e Trabalhadores do SUS.4ª ed., Série B. Textos Básicos de Saúde. Brasília, DF, 2010. Disponível em: http://bibliotecadigital.puc-campinas.edu.br/services/e-books/ humanizasus_documento_gestores_trabalhadores_sus.pdf._Acesso em: 16 jul. 2016.

BRASIL. Ministério da Saúde. Secretaria-Executiva. Núcleo Técnico da Política Nacional de Humanização. HumanizaSUS: acolhimento com avaliação e classificação de risco: um 
paradigma ético-estético no fazer em saúde. 2. ed. Brasília, DF, 2004.

CARDOSO, N. D.; PEREIRA, A. V. Práticas interdisciplinares de acolhimento, educação em saúde e avaliação pós-parto em grupo de puérperas. Revista de Atenção Primária a Saúde, v. 13, n. 4, p. 421-431, 2010.

CARNEIRO, A. C.; OLIVEIRA, A. C. M.; SANTOS, M. M. S.; ALVES, M. S.; CASAIS, N. A.; SANTOS, J. E. Saúde Mental e Atenção Primária: uma experiência com agentes comunitários de saúde em Salvador-BA. Rev. Bras. Prom. Saúde, v.22, n.4, p.264-71, 2009.

CHIAVERINI, D. H.; GONÇALVES, D. A.; BALLESTER, D.; TÓFOLI, L. F; CHAZAN, L. F.; ALMEIDA, N.; FORTES, S. (Org.). Guia prático de matriciamento em saúde mental. Brasília: Ministério da Saúde, 2001. p. 236.

COELHO, E. A. C.; SILVA, C. T. O.; Oliveira, J. F. D.; \& ALMEIDA, M. S. Integralidade do cuidado à saúde da mulher: limites da prática profissional. Anna Nery Revista de Enfermagem, v.13, n.1, p. 154-60, 2009.

COSTA, A. M. Integralidade na atenção e no cuidado a saúde. Saúde e Sociedade, v.13, n.3, p. $5-15,2004$.

CZERESNIA, D. O conceito de saúde e a diferença entre promoção e prevenção. In: CZERESNIA,D.;FREITAS, C. M. (Orgs.).Promoção da Saúde: conceitos, reflexões, tendências. Rio de Janeiro: Ed. Fiocruz, 2003.

DIMENSTEIN, M.; SEVERO, A. K.; BRITO, M.; PIMENTA, A. L.; MEDEIROS, V.; BEZERRA, E. O apoio matricial em unidades de saúde da família: experimentando inovações em saúde mental. Saúde e Sociedade, São Paulo, v. 8, n. 1, p. 63-74, 2009.

FIGUEIREDO, W. Assistência à saúde dos homens: um desafio para os serviços de atenção primária. Ciência \& Saúde Coletiva, v. 10, n. 1, p. 105-109, 2005.

FONTOURA, R. T;; MAYER, C. N. Uma breve reflexão sobre a integralidade. Revista Brasileira de Enfermagem, v. 59, n. 4, p. 532-537,2006.

FRANCO, T.; BUENO, W.; MERHY, E. O acolhimento e os processos de trabalho em saúde: o caso de Betim, Minas Gerais, Brasil. Cad. Saúde Pública, Rio de Janeiro, v. 15, n. 2, p. 345353, abr./jun.1999.

GUANASE, C.; JAPUR, M. Fatores terapêuticos em um grupo de apoio para pacientes psiquiátricos ambulatoriais. Revista Brasileira de Psiquiatria, São Paulo, v. 23, n. 3, p. 134140, 2001.

GUIMARÃES, A. C. P. C. Grupoterapia em Hospital-dia: uma análise temática de quinze sessões. [Dissertação de mestrado não publicada]. Ribeirão Preto, SP: Universidade de São Paulo, 2001.

IBANEZ, G.; MERCEDES, B. P. C.; VEDANAL, K. G. G.; MIASSO, A. I. Adesão e dificuldades relacionadas ao tratamento medicamentoso em pacientes com depressão. Revista Brasileira de Enfermagem, v. 67, n. 4, p. 556-62, 2014. 
JORGE, M. S. B.; PINTO, D. M.; QUINDERÉ, P. H. D., PINTO, A. G. A., DE SOUSA, F. S. P.; CAVALCANTE, C. M. Promoção da Saúde Mental-Tecnologias do Cuidado: vínculo, acolhimento, co-responsabilização e autonomia. Ciência\& Saúde Coletiva, v. 16, n. 7, p. 3051-3060, 2011.

KLEBA, M. E.; WENDHAUSEN, Á. Empoderamento: processo de fortalecimento dossujeitos nos espaços de participação social e democratização política. Saúde e Sociedade, Riode Janeiro, v.18, n.4, p. 733-743, 2009.

KUBARA, C. T. M.; SALES, P. R. S.; MARIN, M. J. S.; TONHOM, S. F. R. Education and health services integration: An integrative review of the literature. Revista Mineira de Enfermagem,v.18, n. 1, p. 207-207, 2014.

NEVES, H.G.; LUCCHESE, R.; MUNARI, D.B. Saúde mental na atenção primária: necessária contribuição de competências. Rev. Bras. Enferm., v.63, n.4, p.666-70, 2010.

PICHON-RIVIÈRE, E.0 processo grupal. São Paulo: Martins Fontes, 2005(Original publicado em 1983).

PINHEIRO, R.S.; VIACAVA, F.; TRAVASSOS, C.; BRITO, A.S. Gênero, morbidade, acesso e utilização de serviços de saúde no Brasil. Ciência \& Saúde Coletiva, Rio de Janeiro, v.7, n.4, p. 687-707, 2002.

ROMBALDI, A. J.; M. C.; GAZALLEI, F. K.; AZEVEDO, M. R.; HALLAL, P. C. Prevalência e fatores associados a sintomas depressivos em adultos do sul do Brasil: estudo transversal de base populacional.Rev. Bras. Epidemiol., SãoPaulo, v. 13, n. 4, p. 620-629, Dec. 2010.

SALINAS, P.; SANTOS, M.A. Serviço de triagem em clínica-escola de Psicologia: A escuta analítica em contexto institucional. Psychê, v. 6, n. 9, p. 177-196, 2002.

SALVENDY, J. T. Seleção, preparação dos pacientes e organização do grupo. In:KAPLAN, H. I.;SADOCK,B. J. (Edit.).Compêndio de Psicoterapia de Grupo.Porto Alegre: Artes Médicas, 1996. p. 63-72.

SEMINOTTI, N. Quebrando paradigmas na concepção dos pequenos grupos: um sistema de sistemas para enfrentar a complexidade humana. In: Pequeno grupo como um sistema complexo: uma estratégia inovadora para produção de saúde na atenção básica. Porto Alegre: Rede UNIDA, 2016a.p. 17-56.

SEMINOTTI, N. Plurivox: Um programa de capacitação para facilitação de grupos na Atenção Básica. In: Pequeno grupo como um sistema complexo: uma estratégia inovadora para produção de saúde na atenção básica. Porto Alegre: Rede UNIDA, 2016b.p. 17-56.

SILVEIRA, L. M. O. B.; GRZYBOWSKI, L. S.; GOMES, R. S.; GADEGAST, S. G. Grupo de acolhida em saúde mental: uma avaliação participante. In: SEMINÁRIO DE EXTENSÃO UNIVERSITÁRIA DA REGIÃO SUL, 32., Resumo ampliado... Paraná, 2014.

SILVEIRA, L.M. C.; RIBEIRO, V. M. B. Grupo de adesão ao tratamento: espaço de "ensinagem" para profissionais de saúde e pacientes. Interface - Comunic., Saúde, Educ., v.9, n. 16, p. 91-104, 2005. 
SOARES, M.H. A inserção do enfermeiro psiquiátrico na equipe de apoio matricial em saúde mental. SMAD - Rev. Eletr. Saude Mental Álc. Drog., v.4, n.2, 2008. Disponível em: http://pepsic.bvsalud.org/pdf/smad/ v4n2/v4n2a06.pdf. Acesso em: 16 jul. 2016.

TEIXEIRA, R. R. O acolhimento num serviço de saúde entendido como uma rede de conversações. Construção da integralidade: cotidiano, saberes e práticas em saúde,v. 3, n. 1, p. 89-111, 2003.

VIEIRA, L. M.; SGAVIOLI, C. A. P. P.; SIMIONATO, E. M. R. S.; INOUE, E. S. Y.; HEUBEL, M. T. C. D.; CONTI, M. H. S.; SAES, S. O.Formação profissional e integração com a rede básica de saúde. Trabalho, Educação e Saúde, Rio de Janeiro, v.14, n.1, p. 293-304, jan./mar. 2016.

VINOGRADOV, S.; YALOM, I. D. Manual de Psicoterapia de Grupo. Porto Alegre: Artes Médicas, 1992.

ZIMERMAN, D. E.; OSÓRIO, L. C. Como trabalhamos com grupos. Porto Alegre: Artmed, 1997. 